\title{
The Impact of Temperature Variations on Spectroscopic Calibration Modelling: A Comparative Study
}

\author{
Tao Chen and Elaine Martin* \\ School of Chemical Engineering and Advanced Materials, \\ University of Newcastle, Newcastle upon Tyne, NE1 7RU, U.K.
}

(Submitted to Journal of Chemometrics, special issue for International Chemometrics Research Meeting (ICRM) 2006)

\begin{abstract}
Temperature fluctuations can have a significant impact on the repeatability of spectral measurements and as a consequence can adversely affect the resulting calibration model. More specifically, when test samples measured at temperatures unseen in the training data set are presented to the model, degraded predictive performance can materialize. Current methods for addressing the temperature variations in a calibration model can be categorized into two classes calibration model based approaches, and spectra standardization methodologies. This paper presents a comparative study on a number of strategies reported in the literature including partial least squares (PLS), continuous piecewise direct standardization (CPDS) and loading space standardization (LSS), in terms of the practical applicability of the algorithms, their implementation complexity, and their predictive performance. It was observed from the study that the global modelling approach, where latent variables are initially extracted from the spectra using PLS, and then augmented with temperature as the independent variable, achieved the best predictive performance. In addition, the two spectra standardization methods, CPDS and LSS, did not provide consistently enhanced performance over the conventional global modelling approach, despite the additional effort in terms of standardizing the spectra across different temperatures. Considering the algorithmic complexity and resulting calibration accuracy, it is concluded that the global modelling (with temperature) approach should be first considered for the development of a calibration model where temperature variations are known to affect the fundamental data, prior to investigating the more powerful spectra standardization approaches.
\end{abstract}

Key words: Spectral standardization; Multivariate spectroscopic calibration; Temperature variation.

\footnotetext{
* Corresponding author. E-mail: e.b.martin@ncl.ac.uk; Tel.: +44 191222 6231; Fax: +44 191222 5292.
} 


\section{Introduction}

As powerful analytical tools, spectroscopic techniques in combination with calibration models have seen increasing implementation in sectors as diverse as food, pharmaceuticals and petrochemical [13]. However, as a consequence of the large number of spectral wavelengths, multivariate calibration methods, such as partial least squares (PLS) [4][5] and other approaches including neural networks [1][6] and Gaussian process models [7], are required for the development of robust calibration models. One challenge faced in industrial on-line and in-line applications of spectroscopy is that the samples are not analyzed under well-controlled laboratory conditions, materializing in fluctuations in some of the external factors, such as temperature and pressure. This behaviour will result in significant spectral variations occurring for the same sample under different conditions [8-13], causing the resultant calibration model to perform poorly, if these variations are not taken into account.

The approaches for addressing temperature fluctuations can be categorized into three classes [8]: variable selection methods, calibration model based approaches, and spectra standardization techniques. With a view to eliminating the impact of temperature on the spectra, variable selection techniques can be applied to select those variables (wavelengths) that are less affected by the temperature variations [11][12][14]. However the variable selection strategies typically involve complex selection algorithms and additional computational costs. It was also demonstrated [11][14] that it can be difficult to identify and remove completely those wavelengths affected by temperature, and thus the application of variable selection techniques is potentially limited in terms of applicability.

Within the category of calibration model based approaches, Wülfert et al. [13] proposed to implicitly include temperature into the calibration design by pooling available spectra under different temperatures to train a PLS model. Alternatively the temperature can be explicitly included into the PLS model as an additional variable; however such methods were found to provide inferior prediction results compared with the implicit method, the rationale being the nonlinear effect of temperature on the spectra [11]. To account for the non-linear effect of temperature, Hageman et al. [8] reviewed several non-linear regression methods, including locally weighted regression [15], neural networks [15], penalized signal regression [16][17] and support vector machine [18][19], where support vector machine achieved the most promising prediction performance. In [20], a parallel factor analysis (PARAFAC) model is used to extract and separate 
source of temperature-related information in a system, and the PARAFAC sample scores were used to build a multiple linear regression (MLR) model for calibration.

The alternative class of techniques to correct for the temperature fluctuations are based on the standardisation of the spectral measurement so that they appear to have been recorded at the same temperature. Piecewise direct standardization (PDS) [21][22] was proposed for the development of calibration models where the measurements are obtained under a discrete set of conditions (e.g. through the utilisation of a number of instruments or different but specific temperature values). To extend the concept of PDS to continuous external factors, such as temperature as observed in an online or at-line industrial application, the continuous piecewise direct standardization (CPDS) algorithm was proposed [23]. More recently, loading space standardization (LSS) [24] was proposed where the standardization is implemented in the loading space derived from the data, as opposed to the original data space. These approaches are explored in more detail in $\S 2.3$ and $\S 2.4$. Another related approach is orthogonal signal correction [25] where variation in the spectra that is not relevant to the prediction is removed, and as a result the temperature-induced variation may be eliminated.

As a consequence of the rapid development of temperature-corrected calibration models, a rigorous assessment and comparison of a number of the available techniques is required, and forms the basis of this paper. In 2000, Wülfert et al. [23] undertook a comparison of the predictive performance of a number of temperature correction techniques against which they benchmarked their proposed CPDS method. A more comprehensive study on temperature robust calibration was carried out by Hageman et al. [8]. However, a limitation of these studies was that in the experimental section, the training samples measured under all available temperatures were used for standardization, and thus the temperatures of the test samples have already been seen in the training stage. In practice, in real applications, both the test samples and test temperature values may not have been "seen" in the training stage. For example, for in-line and on-line measurement of process variables using spectroscopy, the process temperatures will change as a consequence of the control strategy and operational conditions hence the test temperature values may have been unseen in the calibration stage. To investigate this scenario, this paper evaluates the predictive performance of the resulting calibration models by excluding those training spectra recorded at the same temperature as the test samples. In reality, this is the worst case scenario. Techniques considered in this study include local and global models, and two spectral standardization approaches, i.e. CPDS and LSS. The discussion is restricted to linear methods since CPDS and LSS were originally applied to linear PLS models. 
The comparison focuses on the issues of practical applicability, implementation complexity, and predictive performance. The methods are evaluated on a benchmark near infrared data set previously utilized for the evaluation of methodologies for the correction of temperature variations [11], and a mid infrared data set collected for the purpose of the on-line prediction of the concentration of L-glutamic acid (LGA) within water solutions.

\section{Theory}

The following PLS regression model [4][5] forms the basis for the multivariate calibration analysis undertaken in the paper:

$$
\mathbf{Y}=\mathbf{X B}+\mathbf{E}
$$

where $\mathbf{Y}_{n \times h}$ is a matrix of response variables to be predicted where $n$ is the number of spectra and $h$ is the number of response variables, $\mathbf{X}_{n \times p}$ is a matrix of predictor variables where $p$ is the number of wavelengths, $\mathbf{B}$ is the matrix of regression coefficients and $\mathbf{E}$ is the regression residual matrix.

\subsection{Local modelling approach}

The method of local modelling, where separate PLS models are built for each subgroup of temperature values, is first considered. Under well-controlled laboratory conditions, experiments can be designed to record the spectra of the same chemical sample at different temperatures. Let $\mathbf{X}\left(t_{1}\right), \mathbf{X}\left(t_{2}\right), \ldots, \mathbf{X}\left(t_{K}\right)$ be the spectra for the same set of training samples measured at temperatures $t_{1}, t_{2}, \ldots, t_{K}$, from which $K$ PLS models are obtained. Each $\mathbf{X}\left(t_{k}\right)(k=1, \ldots, K)$ is a matrix of order $(n \times p)$. The response of the unseen data is predicted by using the local model whose temperature value is the closest to that of the unseen data.

Consequently the performance of the local modelling approach is strongly dependent on the "temperature resolution", that is, the number of temperature values, i.e. $K$, at which the spectra is recorded for each training sample. When the test sample is measured at the same temperature as one of the local models, excellent results are expected since this situation essentially removes the temperature variation inherent within the spectra. However, if the test sample is measured at a 
temperature that significantly differs from those used for training, the local model approach is expected to give poor predictive performance. The other issue with local modelling is that well designed experiments are necessary to ensure that for each temperature value, a sufficiently large number of samples, $n$, are analyzed to provide enough data for the construction of a robust PLS calibration model. This strategy incurs both significant costs and is time consuming.

In this comparative study, local models provide a lower bound for the prediction error where the test sample has been measured at a temperature that has been presented during the training stage.

\subsection{Global modelling approach}

A global PLS model is obtained by pooling the available training samples measured at the different temperatures and applying PLS to the concatenated matrix. It has been observed [11] that global models are capable of capturing the temperature variations by including more latent variables than that required for the individual local models, and can provide acceptable prediction performance. This approach is referred to as "Global_1" in this study and is illustrated in Figure 1 (a).

(Figure 1 about here)

Intuitively, incorporating the spectra with the temperature value as a predictor should theoretically improve the predictive ability of the calibration model, since information pertaining to the temperature is incorporated in the analysis. However it was reported [11] that by appending temperature to the spectra for PLS modelling, prediction performance is degraded. This methodology is referred to as "Global_2" in this study and is illustrated in Figure 1 (b).

Wülfert et al. [11] also investigated two approaches whereby temperature is used as the response variable. The first approach was based on the appending of the temperature variable to the matrix of response variables to form the $\mathbf{Y}$ block and then PLS2 modelling is implemented. The rationale behind this approach was that through the simultaneous prediction of the temperature and the analyte properties, the model had the ability to identify the temperature-dependent spectral regions. The second methodology proposed was based on a two step modelling approach. In step one, a PLS model is built between the training spectra ( $\mathbf{X}$ block) and the temperature ( $\boldsymbol{y}$ block) and one latent variable is retained. It was hypothesised that the first step removes the temperature effect. Then in step two, the $\mathbf{X}$ residuals from the first PLS model are used to develop the calibration 
model for predicting the analyte properties. The two approaches appear conceptually attractive, but neither of them were observed to give better results than the conventional global model approach ("Global_1") [11]. Therefore they are not considered further in this study.

The lack of improvement in the predictive ability of the resulting calibration model developed through the appending of temperature to the spectra may be due to the fact that the number of wavelengths is so large that they mask the impact of the one-dimensional temperature variable when calculating the PLS latent variables. Hence it may be advantageous if the latent variables are first extracted from the spectra, and then are augmented with the temperature variable which serves as another predictor variable. The idea is analogous to that of the multi-block PLS algorithm [26], where separate block models are built for the spectral and process data (e.g. temperature and pressure), and then these multiple models are combined. However in the context of temperaturerobust calibration, the block of "process data" consists of only one variable, i.e. the temperature, and hence the temperature is directly appended to the extracted latent variables for future regression analysis. This is in contrast to combining these inherently multi-block measurements directly for PLS modelling as in the "Global_2" model. More specifically a two-step approach is proposed. Firstly a conventional "Global_1" model is built to extract the latent variables from the spectra. Then the latent variables are combined with the temperature to build another regression model for predicting $\mathbf{Y}$. In the second step the regression is implemented using a multiple linear regression (MLR) model since typically the dimension of the latent variables is sufficiently low. This model is referred to as "Global_3" and is illustrated in Figure 1 (c).

\subsection{Continuous piecewise direct standardization}

Piecewise direct standardization (PDS) [21][22] was originally devised for the development of spectroscopic calibration models under different sets of conditions. As PDS is only suitable for standardization under a discrete set of conditions, continuous piecewise direct standardization (CPDS) [23] was proposed to account for the variation in the spectra resulting from continuous external factors, such as temperature.

The first step in the PDS and CPDS algorithms is to construct a PLS model between the spectra, $\mathbf{X}\left(t_{\text {ref }}\right)$ which is the order of $(n \times p)$, and the response matrix, $\mathbf{Y}$, for a specific reference 
temperature, $t_{\text {ref }}$. According to the PDS algorithm, a matrix is then calculated that transforms the spectral measurements recorded at temperature values other than at $t_{\text {ref }}$ :

$\mathbf{X}\left(t_{\text {ref }}\right)=\mathbf{X}\left(t_{k}\right) \mathbf{Q}\left(t_{k}\right), \quad\left(k=1,2, \ldots, K ; t_{k} \neq t_{\text {ref }}\right)$

where $\mathbf{Q}\left(t_{k}\right)$ is a $(p \times p)$ banded transformation matrix that is obtained by linearly regressing the $j$-th column of $\mathbf{X}\left(t_{\text {ref }}\right)$ on a sub-matrix taken from $\mathbf{X}\left(t_{k}\right)$ (i.e. the spectra in $\mathbf{X}\left(t_{k}\right)$ corresponding to a window of wavelengths from $j$-w to $j+w$ ), using PLS, where the window size, $2 w+1$, is determined by the tuning parameter w. $\mathbf{Q}\left(t_{k}\right)$ is then formed by placing the regression vectors on corresponding blocks [23]. This procedure is repeated for each temperature value $t_{k},\left(k=1,2, \ldots, K ; t_{k} \neq t_{\text {ref }}\right)$, and $K$ transformation matrices are obtained by noting that $\mathbf{Q}\left(t_{\text {ref }}\right)$ is an identity matrix.

To account for the effect of continuous temperature values, a polynomial function is fitted against the temperature difference $\Delta t_{k}$, where $\Delta t_{k}=t_{k}-t_{\text {ref }}$, to the non-zero elements of the banded matrix $\mathbf{Q}\left(t_{k}\right)$ :

$q_{i j}\left(t_{k}\right)=a_{i j}+b_{i j} \Delta t_{k}+c_{i j} \Delta t_{k}^{2}+e_{i j}$

where $q_{i j}\left(t_{k}\right)$ is the element of $\mathbf{Q}\left(t_{k}\right)$ for the $i$-th row and $j$-th column. Once the parameters of the polynomial function have been estimated, the transformation matrix for the spectra measured at an unseen temperature value, $t_{\text {test }}$, in the test stage can be calculated by applying Eq. (3). Hence the temperature influence can be removed by transforming the spectral matrix as if it were measured at the reference temperature:

$$
\mathbf{X}\left(t_{\text {ref }} \mid t_{\text {test }}\right)=\mathbf{X}\left(t_{\text {test }}\right) \mathbf{Q}\left(t_{\text {test }}\right)
$$

The responses can then be predicted by the PLS model built under the reference temperature.

One of the main issues with CPDS, as identified by Chen et al. [24], is the need to determine a number of tuning parameters. Apart from the number of latent variables to include in the PLS 
calibration model for the reference temperature, there are three additional parameters to be determined: the width of the band in $\mathbf{Q}\left(t_{k}\right)(2 \mathrm{w}+1)$; the number of latent variables in the PLS model to obtain $\mathbf{Q}\left(t_{k}\right)$; and the order of the polynomial. It was proposed that these tuning parameters are determined by using cross-validation [23][27]. Although the polynomial is typically taken to be second order by appealing to the established findings in the literature [24][28][29], the application of cross-validation to attain the other tuning parameters is a time-consuming process.

\subsection{Loading space standardization}

The main idea behind the loading space standardization (LSS) approach [24] is to perform standardization on the loading space, as opposed to the original data space. The philosophy being that the loading space may be a more sparse and robust representation of the original data, and hence may provide more reliable standardization results. More specifically as for CPDS, LSS requires the selection of a reference temperature, $t_{\text {ref }}$, and a PLS model is then developed based on the spectra collected at this reference temperature. To obtain the loading space of the original data, the average spectra across different temperatures are first calculated: $\overline{\mathbf{X}}=\sum_{k=1}^{K} \mathbf{X}\left(t_{k}\right) / K$, to which singular value decomposition (SVD) is applied:

$\overline{\mathbf{X}}=\overline{\mathbf{T}} \mathbf{P}^{\mathrm{T}}+\mathbf{E}$

where $\mathbf{P}$ is the SVD loading matrix, $\overline{\mathbf{T}}$ is the corresponding score matrix, and $\mathbf{E}$ is the residual term due to discarding those SVD factors associated with small singular values. For temperature $t_{k}$, the spectra can be represented as:

$$
\mathbf{X}\left(t_{k}\right)=\overline{\mathbf{T}} \mathbf{P}\left(t_{k}\right)^{\mathrm{T}}+\mathbf{E}\left(t_{k}\right)
$$

Ignoring the residual term, the loading matrix is given by $\mathbf{P}\left(t_{k}\right)^{\mathrm{T}} \approx \overline{\mathbf{T}}^{+} \mathbf{X}\left(t_{k}\right)$, where the superscript " + " is the Moore-Penrose matrix inverse. In the next step, a second order polynomial of the temperature $t_{k}(k=1, \cdots K)$ (as opposed to the temperature difference in CPDS in Eq. (3)) can be fitted to the elements of $\mathbf{P}\left(t_{k}\right)$ : 
$p_{i j}\left(t_{k}\right)=a_{i j}+b_{i j} t_{k}+c_{i j} t_{k}^{2}+e_{i j}\left(t_{k}\right)$

After the polynomial coefficients, $a_{i j}, b_{i j}$ and $c_{i j}$, have been estimated by minimizing the sum of squared error, the loading matrix $\mathbf{P}\left(t_{\text {test }}\right)$ for an unseen test temperature $t_{\text {test }}$ can be calculated (Eq. (7)). The score matrix $\mathbf{T}$ of the spectra recorded at this test temperature, $\mathbf{X}\left(t_{\text {test }}\right)$, can then be estimated as $\mathbf{T}=\mathbf{X}\left(t_{\text {test }}\right)\left(\mathbf{P}\left(t_{\text {test }}\right)^{\mathrm{T}}\right)^{+}$, and can be used to transform $\mathbf{X}\left(t_{\text {test }}\right)$ so that it appears to have been recorded under the reference temperature:

$\mathbf{X}\left(t_{\text {ref }} \mid t_{\text {test }}\right)=\mathbf{T}\left(\mathbf{P}\left(t_{\text {ref }}\right)-\mathbf{P}\left(t_{\text {test }}\right)\right)^{\mathrm{T}}+\mathbf{X}\left(t_{\text {test }}\right)$

The transformed spectra can then be used for the prediction of the target values using the calibration model developed under the reference temperature.

LSS has three tuning parameters, i.e. the number of latent variables in the PLS model for the reference temperature, the number of SVD factors retained, and the order of the polynomial, while in CPDS four parameters are required to be tuned. In this sense, the implementation of LSS is slightly more straightforward than that of CPDS.

\section{Comparative study}

\subsection{Data set}

Two sets of spectroscopic data are considered in this comparative study. Data set A was presented in Wülfert et al. [11], and comprises 95 near infrared (NIR) spectra of 19 ternary mixtures of ethanol, water, and 2-propanol, which were recorded in the range $580-1049 \mathrm{~nm}$ with a resolution of $1 \mathrm{~nm}$ using a HP 8454 spectrophotometer, equipped with a thermostable cell holder at five temperatures $\left(30,40,50,60\right.$, and $\left.70{ }^{\circ} \mathrm{C}\right)$. The spectral region between 749 and $849 \mathrm{~nm}$ was used for slope and offset correction, and the 200 absorbance values in the range 850-1049 $\mathrm{nm}$ defined the area of interest for the calibration model. Experimental details of this data set are described in [11], and the data is available at http://www.bdagroup.nl/downloads/bda_downloads.html. 
Data set B was collected to determine the concentration of L-glutamic acid (LGA) within water solutions. It comprises 45 Fourier transform infrared spectra recorded using an attenuated total reflection probe (ATR-FTIR) for 13 samples with different LGA concentrations measured at different temperature values. The spectra covered the range $400-4000 \mathrm{~cm}^{-1}$ with a resolution of 4 $\mathrm{cm}^{-1}$. The spectral region $700-2000 \mathrm{~cm}^{-1}$ was used for the development of a calibration model. The calibration experimental matrix is given in Table 1. The ultimate objective for collecting this data set was to implement an on-line LGA concentration sensor for the control of the super-saturation during the crystallization of LGA [30]. The temperature effect on the spectra must be taken into consideration when developing a calibration model, since the crystallization process includes a heating and cooling stage which introduces a large temperature change. Hence the data were collected across the temperature range $40-90{ }^{\circ} \mathrm{C}$. Furthermore some of the spectra were recorded in the concentration region close to the solubility value, where the onset of the crystallization process potentially occurs.

(Table 1 about here)

\subsection{Evaluation}

This study compares the temperature-corrected calibration models, described in Section 2, in terms of their practical applicability, algorithmic complexity, and predictive performance. The values of the tuning parameters for CPDS and LSS are determined using cross validation. To obtain a reliable estimate of the predictive performance, defined in terms of the root mean squared error for prediction (RMSEP), the test samples must have been unseen in the training data set used for the development of the temperature correction method and the calibration model. The term "unseen" defines two aspects, firstly an unseen temperature value, i.e. the training data set should not include spectra recorded at the same temperature as those of the test samples; and secondly it relates to unseen test samples, i.e. the spectra from the same sample cannot be used in both the training and testing stage, even though they may be recorded at different temperatures. The requirement for an unseen temperature value is important for an accurate estimate of the predictive performance since in practical on-line and in-line industrial applications, the spectra of the samples being measured are typically recorded at temperatures not necessarily seen in the training stage. For example in the case of data set $\mathrm{B}$, the temperature profile during a crystallization process changes and the temperature values will not necessarily have been incorporated in the training data set. By adopting this 
methodology of evaluation, the results presented in this paper for data set A are different from those obtained by Wülfert et al. [11][23].

For data set A, the 19 samples at each temperature were divided into 13 training samples and 6 test samples according to the original paper [11]. Data set B comprises 13 samples, which is not sufficient to enable a separate training and test set to be defined. The strategy adopted in this study was to first select one sample recorded at a temperature value to define the test sample, with the remaining samples measured at different temperature forming the training data set. This procedure is executed for each sample under each temperature in data set B, and the average RMSEP is used as the criterion for comparison.

\section{Results and discussions}

\subsection{Practical applicability}

As discussed in Section 2.1, the applicability of the local modelling approach depends on the "temperature resolution", i.e. the number of temperature values at which the spectra of the training samples were recorded. If a sufficient number of temperature values are available to develop local models, it is likely that the test samples will have been measured under a temperature close to the temperature associated with one of the local models. Therefore the effect of temperature on the spectra will be minimized. However, to analyze the same set of samples for a large number of temperature values is both time-consuming and costly thereby introducing additional time and costs, and thus its application is potentially limited. In contrast, the methodology of global modelling, including the three global models described in Section 2.2, is applicable when the training data set is limited and the local modelling approach cannot be applied.

The spectra standardization methods of CPDS and LSS require the same sample to be analyzed for a number of different temperature values. The number of values must be sufficiently large to realize the fitting of a polynomial function. For example, a second order polynomial function requires at least three different temperature values, since three parameters require to be determined. In practice, this requirement may not be satisfied. For example, as can be observed from data set B (Table 1) not all the samples were analyzed under the same set of temperature values. This issue is not a result of the failure to implement experimental design but it is a consequence of the inherent characteristics of the system. For example in practice it is not possible to prepare a solution with a 
LGA concentration of $10.00\left(\mathrm{~g} / 500 \mathrm{ml}\right.$ water) for a temperature of $40^{\circ} \mathrm{C}$, since the maximum concentration at this temperature, i.e. the solubility, is 7.55 . For this data set, if the reference temperature in CPDS, $t_{\text {ref }}$, is selected to be $70^{\circ} \mathrm{C}$, only two samples were measured at both 40 and $70^{\circ} \mathrm{C}$. Hence the transformation matrix, $\mathbf{Q}\left(t_{k}\right)$, cannot be reliably obtained by regressing the spectra of the same samples at for these two different temperatures. This example presents even more serious problems for LSS, since LSS requires that all the samples are analyzed for all temperature values in order to calculate the average spectra. Thus LSS is not applicable in this case study.

\subsection{Implementation complexity}

The global modelling approaches materialize in a single global model in contrast to the multiple models that are developed if the local modelling approach is adopted. However, the global models require the retention of additional latent variables compared with the local models to explain the additional variability materializing from the temperature variations. In comparison with conventional PLS, the spectra standardization approaches require the tuning of additional factors using cross-validation. For example, there are three tuning factors in CPDS to be determined: the width of the band in $\mathbf{Q}\left(t_{k}\right)(2 w+1)$, the number of latent variables in the PLS modelling algorithm to obtain $\mathbf{Q}\left(t_{k}\right)$, and the order of the polynomial function, whilst in LSS, the number of latent factors retained in the SVD algorithm and the order of the polynomial function require to be determined. Although the polynomial function is typically taken to be second order by appealing to the established findings in the literature [24][28][29], the cross-validation of the other tuning parameters is still a time-consuming process.

The other issue when implementing the spectra standardization approaches is to determine the optimal reference temperature. It was suggested [23] that the reference temperature should be selected to be close to the value at which the test samples were analyzed. However this may not necessarily be optimal. Based on data set A, Figure 2 compares the RMSEP when two reference temperatures $\left(30\right.$ and $\left.50^{\circ} \mathrm{C}\right)$ are selected for the prediction at temperature $70^{\circ} \mathrm{C}$. For both CPDS and LSS, the results do not consistently conclude that using $50^{\circ} \mathrm{C}$ as the reference is better than using $30^{\circ} \mathrm{C}$, despite the fact that $50^{\circ} \mathrm{C}$ is closer than $30^{\circ} \mathrm{C}$ to the test temperature. Hence the selection of the reference temperature requires more advanced techniques, for example cross-validation, which makes the implementation of the spectra standardization approaches more complex. 
(Figure 2 about here)

\subsection{Predictive performance}

The predictive performance of the calibration models for data set A is presented in Table 2, 3 and 4 . Table 2 compares the RMSEP of CPDS and LSS under different reference and test temperature values. It can be seen from Table 2 that for both CPDS and LSS, the prediction errors for the test samples at 40,50 and $60^{\circ} \mathrm{C}$ are satisfactory, and are generally lower than those for the test samples at 30 and $70{ }^{\circ} \mathrm{C}$. This can be explained by the fact that 30 and $70{ }^{\circ} \mathrm{C}$ are the lowest and highest temperatures in the data set, and thus the extrapolation errors are higher than the interpolation errors. This phenomenon has been noted by Chen et al. [24]. The other finding from Table 2 is that, although CPDS and LSS achieved different RMSEPs at different reference and test temperatures, they are observed to provide overall similar predictive performance. For example, if the reference temperature is taken as $50{ }^{\circ} \mathrm{C}$, the RMSEPs averaged over the other test temperatures are similar (see last two rows in Table 4).

(Table 2 about here)

(Table 3 about here)

(Table 4 about here)

Table 3 compares the predictive performance of the local and global models under different temperatures for data set A. The local model approach provides the best result and serves as the lower bound for the RMSEP. However, as discussed in Section 4.1, the local model approach is not applicable if the test temperature is unseen at the training stage and is far from the training temperatures. The "Global_1" model addresses the temperature variations explicitly by pooling all training data at different temperatures for the PLS model, while the "Global_2" model augments the temperature with the spectral variables as the input variables for the PLS regression model. Although the "Global_2" model explicitly considers the impact of temperature variations, it achieves a higher RMSEP than the "Global_1" model. This phenomenon has been observed by Wülfert et al. [11], and it may be a consequence of the fact that the number of spectral variables (wavelengths) is so large that the direct inclusion of temperature does not bring meaningful information into the model. This hypothesis motivated the development of the "Global_3" model where the latent variables (using PLS) from the spectra are augmented with the temperature for a 
second stage linear regression. As the number of latent variables is much smaller than the number of wavelengths, the inclusion of temperature at this stage is expected to be more informative. This can be confirmed by reference to Table 3, where the "Global_3" model achieves a lower RMSEP than the "Global_1" and "Global_2" models in most cases.

Table 4 summarizes the predictive performance of the different calibration models for data set A. Following the suggestion to select a median value in the temperate range [23], the reference temperature for CPDS and LSS is taken as $50^{\circ} \mathrm{C}$, and the RMSEPs are averaged over the test samples under other temperatures for all the calibration models. The conclusions drawn from Table 4 include:

- Temperature variations adversely affect the predictive performance of calibration models, since the local model, where no temperature variation is present, achieves the best result.

- Of the three global models, "Global_3" gives the best result through the incorporation of the temperature information explicitly.

- The two spectra standardization approaches, i.e. CPDS and LSS, are comparable to each other in terms of the RMSEP. In addition, they perform marginally better than "Global_1", but are inferior to the "Global_3".

As discussed in Section 4.1, the spectra standardization methods are not applicable to Data set B, since not all the samples were recorded under the same set of temperature values. Furthermore the local modelling approach cannot be applied due to the limited amount of data under each temperature value. Therefore only the global calibration models are developed, with RMSEPs being 2.23 (“Global_1"), 2.77 (“Global_2") and 1.81 (“Global_3").The results are consistent with those for data set A, with the best results being achieved by the "Global_3" model. Figure 3 presents a plot of the predicted LGA concentration versus the true value for the "Global_1" and "Global_3" models, where the superior result of the "Global_3" model is clearly evident. A summary of the comparative study is given in Table 5 .

(Figure 3 about here)

(Table 5 about here)

\section{Conclusions}


This paper has presented a comparative study of the strategies for the development of a calibration model, where the impact of temperature variations in the spectra measurements is considered. A number of approaches are presented and compared, including local and global partial least squares (PLS) models, and two spectra standardization methods, i.e. continuous piecewise direct standardization (CPDS) and loading space standardization (LSS). The comparison and discussion of these approaches focuses on the issues of practical applicability, implementation complexity, and predictive performance. It was found that the global modelling approach, where latent variables are first extracted from the spectra using PLS, and then augmented with the temperature as an independent variable, achieved the best predictive performance, while the two spectra standardization methods did not provide consistently enhanced performance over the conventional global modelling approach. Furthermore, compared with CPDS and LSS, the global modelling approach has the advantage of being applicable to a wide range of situations and is also of low implementation complexity. Therefore it is concluded that the global modelling (with temperature) approach should be first considered for the development of calibration models with temperature variations, prior to investigating the more powerful spectra standardization approaches.

Future work focuses on the extension of the global modelling approach to address the impact of multiple external factors (e.g. temperature and pressure) on the spectra measurement using multiblock PLS approach. In addition, the concept of modelling the spectra and temperature in a twostage procedure as in the "Global_3" model may be extended to non-linear regression algorithms, e.g. neural networks or support vector machines, and this is a further research direction.

\section{Acknowledgments}

T. Chen would like to acknowledge the financial support from the EPSRC KNOW-HOW (GR/R19366/01) and VERTIGO (GR/R64407/01) and the UK ORS Award for his PhD study. The LGA data (data set B) was provided by the University of Leeds under the project EPSRC "Chemicals Behaving Badly II" (GR/R43853/01).

\section{References}

1. Borggaard C, Thodberg HH. Optimal minimal neural interpretation of spectra. Analytical Chemistry 1992; 64: 545-551. 
2. Antti H, Sjöström M, Wallbäcks L. Multivariate calibration models using NIR spectroscopy on pulp and paper industrial applications. Journal of Chemometrics 1996; 10: 591-603.

3. Dyrby M, Engelsen SB, Norgaard L, Bruhn M, Lundsberg-Nielsen L. Chemometric quantitation of the active substance in a pharmaceutical tablet using near infrared (NIR) transmittance and NIR FT-Raman spectra. Applied Spectroscopy 2002; 56: 579-585.

4. Geladi P, Kowalski BR. Partial least-squares regression: a tutorial. Analytica Chimica Acta 1986; 185: $1-17$.

5. Hoskuldsson A. PLS regression methods. Journal of Chemometrics 1988; 2: 211-228.

6. Thodberg HH. A review of Bayesian neural networks with an application to near infrared spectroscopy. IEEE Transactions on Neural Networks 1996; 7: 56-72.

7. Chen T, Morris J, Martin E. Gaussian process regression for multivariate spectroscopic calibration. Chemometrics and Intelligent Laboratory Systems 2007; in press.

8. Hageman JA, Westerhuis JA, Smilde AK. Temperature robust multivariate calibration: an overview of methods for dealing with temperature influences on near infrared spectra. Journal of Near Infrared Spectroscopy 2005; 13: 53-62.

9. DeBraekeleer K, Cuesta Sanchez F, Hailey PA, Sharp DCA, Pettman AJ, Massart DL. Influence and correction of temperature perturbations on NIR spectra during the monitoring of a polymorph conversion process prior to self-modelling mixture analysis. Journal of Pharmaceutical and Biomedical Analysis 1998; 17: 141-152.

10. Yalvac ED, Seasholtz MB, Beach MA, Crouch SR. Real-time analysis of light alkenes at elevated temperatures and pressures by fiber-optic near-infrared spectroscopy. Applied Spectroscopy 1997; 51: 1565-1572.

11. Wülfert F, Kok WT, de Noord OE, Smilde AK. Linear techniques to correct for temperatureinduced spectral variation in multivariate calibration. Chemometrics and Intelligent Laboratory Systems 2000; 51: 189-200.

12. Swierenga H, Wülfert F, de Noord OE, de Weyer AP, Smilde AK, Buydens LMC. Development of robust calibration models in NIR spectroscopic applications. Analytical Chimica Acta 2000; 411: $121-135$.

13. Wülfert F, Kok WT, Smilde AK. Influence of temperature on vibrational spectra and consequences for the predictive ability of multivariate models. Analytical Chemistry 1998; 70: 1761-1767.

14. Brown PJ. Measurement, Regression and Calibration. Clarendon Press, Oxford, 1993.

15. Despagne F, Massart DL, Chabot P. Development of a robust calibration model for nonlinear in-line process data. Analytical Chemistry 2000; 72: 1657-1665. 
16. Marx BD, Eilers PHC. Multivariate calibration stability: a comparison of methods. Journal of Chemometrics 2002; 16: 129-140.

17. Eilers PHC, Marx BD. Multivariate calibration with temperature interaction using twodimensional penalized signal regression. Chemometrics and Intelligent Laboratory Systems 2003; 66: 159-174.

18. Thissen U, Üstün B, Melssen WJ, Buydens LMC. Multivariate calibration with least-squares support vector machines. Analytical Chemistry 2004; 76: 3099-3105.

19. Thissen U, Pepers M, Üstün B, Melssen WJ, Buydens LMC. Comparing support vector machines to PLS for spectral regression applications. Chemometrics and Intelligent Laboratory Systems 2004; 73: 169-179.

20. Peinado AC, van den Berg F, Blancoa M, Bro R. Temperature-induced variation for NIR tensorbased calibration. Chemometrics and Intelligent Laboratory Systems 2006; 83: 75-82.

21. Wang Y, Veltkamp DJ, Kowalski BR. Multivariate instrument standardization. Analytical Chemistry 1991; 63: 2750-2756.

22. Wang Y, Kowalski BR. Temperature-compensating calibration transfer for near-infrared filter instruments. Analytical Chemistry 1993; 65: 1301-1303.

23. Wülfert F, Kok WT, de Noord OE, Smilde AK. Correction of temperature-induced spectral variation by continuous piecewise direct standardization. Analytical Chemistry 2000; 72: 16391644.

24. Chen ZP, Morris J, Martin E. Correction of temperature-induced spectral variations by loading space standardization. Analytical Chemistry 2005; 77: 1376-1384.

25. Westerhuis JA, de Jong S, Smilde AK. Direct orthogonal signal correction. Chemometrics and Intelligent Laboratory Systems 2001; 56: 13-25.

26. Westerhuis JA, Kourti T, MacGregor JF. Analysis of multiblock and hierarchical PCA and PLS models. Journal of Chemometrics 1998; 12: 301-321.

27. Bärring HK, Boelens HFM, de Noord OE, Smilde AK. Optimizing meta-parameters in continuous piecewise direct standardization. Applied Spectroscopy 2001; 55: 458-466.

28. Lewiner F, Klein JP, Peul F, Fevotte G. On-line ATR FTIR measurement of supersaturation during solution crystallization processes. Calibration and applications on three solute/solvent systems. Chemical Engineering and Science 2001; 56: 2069-2084.

29. Dunuwila DD, Berglund KA. ATR FTIR spectroscopy for in situ measurement of supersaturation. Journal of Crystal Growth 1997; 179: 185-193. 
30. Grön H, Borissova A, Roberts KJ. In-process ATR-FTIR spectroscopy for closed-loop supersaturation control of a batch crystallizer producing monosodium glutamate crystals of defined size. Industrial \& Engineering Chemistry Research 2003; 42: 198-206. 
Table 1: Calibration experimental matrix as a function of temperature and LGA concentration. Cells with the symbol, $\boldsymbol{\Lambda}$, indicate that the data at corresponding temperature and concentration are available.

\begin{tabular}{|c|c|c|c|c|c|c|}
\hline \multirow{2}{*}{$\begin{array}{l}\text { LGA Concentration } \\
\text { (g/500ml water) }\end{array}$} & \multicolumn{6}{|c|}{ Temperature $\left({ }^{\circ} \mathrm{C}\right)$} \\
\hline & 40 & 50 & 60 & 70 & 80 & 90 \\
\hline 1.50 & $\Delta$ & & & & & \\
\hline 3.00 & $\Delta$ & $\Delta$ & & & & \\
\hline 4.50 & $\Delta$ & $\Delta$ & $\Delta$ & & & \\
\hline 6.00 & $\Delta$ & $\Delta$ & $\Delta$ & $\Delta$ & $\Delta$ & $\Delta$ \\
\hline 7.50 & $\Delta$ & $\Delta$ & $\boldsymbol{\Delta}$ & $\boldsymbol{\Delta}$ & $\boldsymbol{\Delta}$ & $\Delta$ \\
\hline 10.00 & & $\boldsymbol{\Delta}$ & $\Delta$ & $\boldsymbol{\Delta}$ & $\boldsymbol{\Delta}$ & $\Delta$ \\
\hline 12.00 & & & $\boldsymbol{\Delta}$ & $\Delta$ & $\boldsymbol{\Delta}$ & $\Delta$ \\
\hline 13.50 & & & $\Delta$ & $\Delta$ & $\Delta$ & $\Delta$ \\
\hline 15.00 & & & $\Delta$ & $\Delta$ & $\boldsymbol{\Delta}$ & $\Delta$ \\
\hline 18.00 & & & & $\Delta$ & $\Delta$ & $\Delta$ \\
\hline 22.50 & & & & $\Delta$ & $\Delta$ & $\Delta$ \\
\hline 27.00 & & & & & $\boldsymbol{\Delta}$ & $\Delta$ \\
\hline 31.25 & & & & & $\Delta$ & $\Delta$ \\
\hline Solubility & 7.55 & 10.95 & 15.85 & 22.95 & 33.30 & 48.30 \\
\hline
\end{tabular}


Table 2: Comparison of CPDS and LSS in terms of RMSEP under different reference and test temperature values for data set $\mathrm{A}$.

\begin{tabular}{|c|c|c|c|c|c|c|c|c|c|c|c|}
\hline \multirow{3}{*}{$\begin{array}{l}t_{\text {ref }} \\
\left({ }^{\circ} \mathrm{C}\right)\end{array}$} & \multirow{3}{*}{$\begin{array}{l}\text { Chemical } \\
\text { Species }\end{array}$} & \multicolumn{10}{|c|}{$t_{\text {test }}\left({ }^{\circ} \mathrm{C}\right)$} \\
\hline & & \multicolumn{2}{|c|}{30} & \multicolumn{2}{|c|}{40} & \multicolumn{2}{|c|}{50} & \multicolumn{2}{|c|}{60} & \multicolumn{2}{|c|}{70} \\
\hline & & CPDS & LSS & CPDS & LSS & CPDS & LSS & CPDS & LSS & CPDS & LSS \\
\hline \multirow[t]{3}{*}{30} & ethanol & & & 0.013 & 0.013 & 0.029 & 0.027 & 0.021 & 0.014 & 0.019 & 0.042 \\
\hline & water & & & 0.007 & 0.006 & 0.016 & 0.016 & 0.016 & 0.009 & 0.027 & 0.055 \\
\hline & 2-propanol & & & 0.013 & 0.010 & 0.033 & 0.028 & 0.018 & 0.010 & 0.017 & 0.014 \\
\hline \multirow[t]{3}{*}{40} & ethanol & 0.016 & 0.012 & & & 0.013 & 0.010 & 0.017 & 0.010 & 0.013 & 0.021 \\
\hline & water & 0.026 & 0.022 & & & 0.015 & 0.013 & 0.007 & 0.007 & 0.017 & 0.039 \\
\hline & 2-propanol & 0.013 & 0.023 & & & 0.020 & 0.017 & 0.017 & 0.011 & 0.022 & 0.031 \\
\hline \multirow[t]{3}{*}{50} & ethanol & 0.031 & 0.013 & 0.009 & 0.011 & & & 0.017 & 0.011 & 0.029 & 0.020 \\
\hline & water & 0.017 & 0.021 & 0.007 & 0.007 & & & 0.006 & 0.006 & 0.013 & 0.027 \\
\hline & 2-propanol & 0.018 & 0.025 & 0.010 & 0.009 & & & 0.013 & 0.010 & 0.018 & 0.024 \\
\hline \multirow[t]{3}{*}{60} & ethanol & 0.029 & 0.012 & 0.013 & 0.009 & 0.014 & 0.014 & & & 0.034 & 0.015 \\
\hline & water & 0.014 & 0.021 & 0.007 & 0.007 & 0.009 & 0.010 & & & 0.026 & 0.018 \\
\hline & 2-propanol & 0.020 & 0.023 & 0.014 & 0.009 & 0.015 & 0.019 & & & 0.023 & 0.020 \\
\hline \multirow[t]{3}{*}{70} & ethanol & 0.020 & 0.012 & 0.017 & 0.007 & 0.012 & 0.015 & 0.014 & 0.009 & & \\
\hline & water & 0.009 & 0.021 & 0.007 & 0.004 & 0.009 & 0.010 & 0.009 & 0.006 & & \\
\hline & 2-propanol & 0.027 & 0.026 & 0.020 & 0.011 & 0.013 & 0.021 & 0.012 & 0.010 & & \\
\hline
\end{tabular}


Table 3: Comparison of local and global models in terms of RMSEP under different test temperature values for data set $\mathrm{A}$.

\begin{tabular}{ccccccc}
\hline \multirow{2}{*}{ Models } & Chemical & \multicolumn{5}{c}{$t_{\text {test }}\left({ }^{\circ} \mathrm{C}\right)$} \\
\cline { 3 - 7 } & Species & 30 & 40 & 50 & 60 & 70 \\
\hline Local & ethanol & 0.018 & 0.011 & 0.017 & 0.010 & 0.011 \\
& water & 0.009 & 0.007 & 0.011 & 0.004 & 0.004 \\
\multirow{2}{*}{ Global_1 } & 2-propanol & 0.012 & 0.009 & 0.022 & 0.008 & 0.015 \\
& ethanol & 0.028 & 0.014 & 0.041 & 0.016 & 0.015 \\
& water & 0.030 & 0.006 & 0.009 & 0.011 & 0.007 \\
Global_2 & 2-propanol & 0.011 & 0.016 & 0.041 & 0.018 & 0.020 \\
& ethanol & 0.030 & 0.012 & 0.032 & 0.018 & 0.035 \\
& water & 0.038 & 0.009 & 0.014 & 0.009 & 0.018 \\
Global_3 & 2-propanol & 0.056 & 0.019 & 0.035 & 0.016 & 0.045 \\
& ethanol & 0.019 & 0.009 & 0.028 & 0.012 & 0.019 \\
& water & 0.025 & 0.007 & 0.012 & 0.008 & 0.007 \\
& 2-propanol & 0.010 & 0.014 & 0.035 & 0.017 & 0.022 \\
\hline
\end{tabular}


Table 4: Comparison of different calibration models for data set A. The RMSEPs are averaged over the test samples under temperatures $30,40,60$, and $70{ }^{\circ} \mathrm{C}$. For CPDS and LSS the reference temperature is taken as $50{ }^{\circ} \mathrm{C}$.

\begin{tabular}{ccccc}
\hline RMSEP & Ethanol & Water & 2-Propanol & Average \\
\hline Local & 0.012 & 0.006 & 0.011 & 0.010 \\
Global_1 & 0.018 & 0.014 & 0.016 & 0.016 \\
Global_2 & 0.019 & 0.017 & 0.028 & 0.021 \\
Global_3 & 0.015 & 0.012 & 0.016 & 0.014 \\
CPDS & 0.021 & 0.011 & 0.014 & 0.015 \\
LSS & 0.014 & 0.015 & 0.015 & 0.015 \\
\hline
\end{tabular}


Table 5: The summary of the comparative study.

\begin{tabular}{|c|c|c|c|}
\hline & Practical Applicability & Implementation Complexity & Predictive Performance \\
\hline Local & $\begin{array}{l}\text { Restricted to small } \\
\text { temperature variations. }\end{array}$ & $\begin{array}{l}\text { Different models for each } \\
\text { local temperature value. }\end{array}$ & The best. \\
\hline Global & $\begin{array}{l}\text { Applicable to a wide range } \\
\text { of situations with large } \\
\text { temperature variations. }\end{array}$ & $\begin{array}{l}\text { One global model with } \\
\text { additional latent variables } \\
\text { compared with local model. }\end{array}$ & $\begin{array}{l}\text { The "Global_3" model is } \\
\text { the best of the global } \\
\text { models, and outperforms } \\
\text { CPDS and LSS. }\end{array}$ \\
\hline CPDS & $\begin{array}{l}\text { Requiring well-designed } \\
\text { experiments to ensure the } \\
\text { same samples being } \\
\text { measured at different } \\
\text { temperatures. }\end{array}$ & $\begin{array}{l}\text { Cross-validation to } \\
\text { optimize three meta- } \\
\text { parameters; Reference } \\
\text { temperature to be } \\
\text { determined. }\end{array}$ & \multirow{2}{*}{$\begin{array}{l}\text { CPDS and LSS are } \\
\text { comparable; } \\
\text { they both outperform } \\
\text { "Global_1" and } \\
\text { "Global_2" models but are } \\
\text { inferior to the "Global_3" } \\
\text { model. }\end{array}$} \\
\hline LSS & The same as CPDS. & $\begin{array}{l}\text { Cross-validation to } \\
\text { optimize two meta- } \\
\text { parameters; Reference } \\
\text { temperature to be } \\
\text { determined. }\end{array}$ & \\
\hline
\end{tabular}


Figure 1: Schematics for global calibration models. (a) Global_1; (b) Global_2; (c) Global_3.

(a)

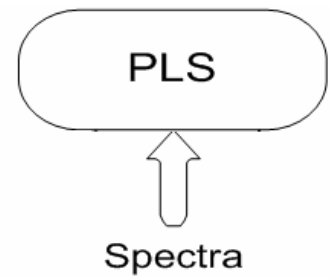

(b)

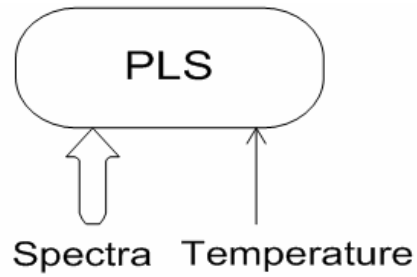

(c)

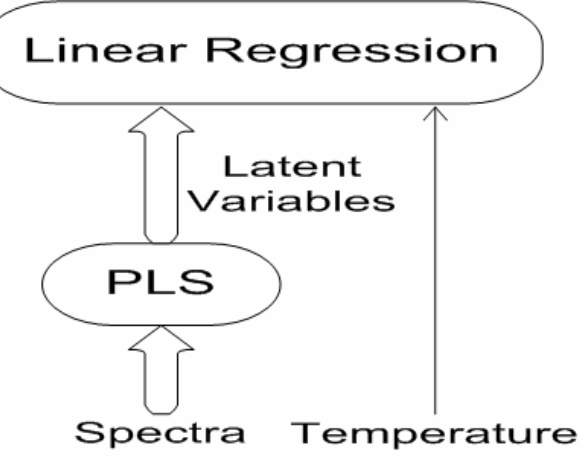


Figure 2: Comparison of different reference temperatures for the prediction at temperature 70 ${ }^{\circ} \mathrm{C}$ for data set A. (a) CPDS; (b) LSS.

(a)

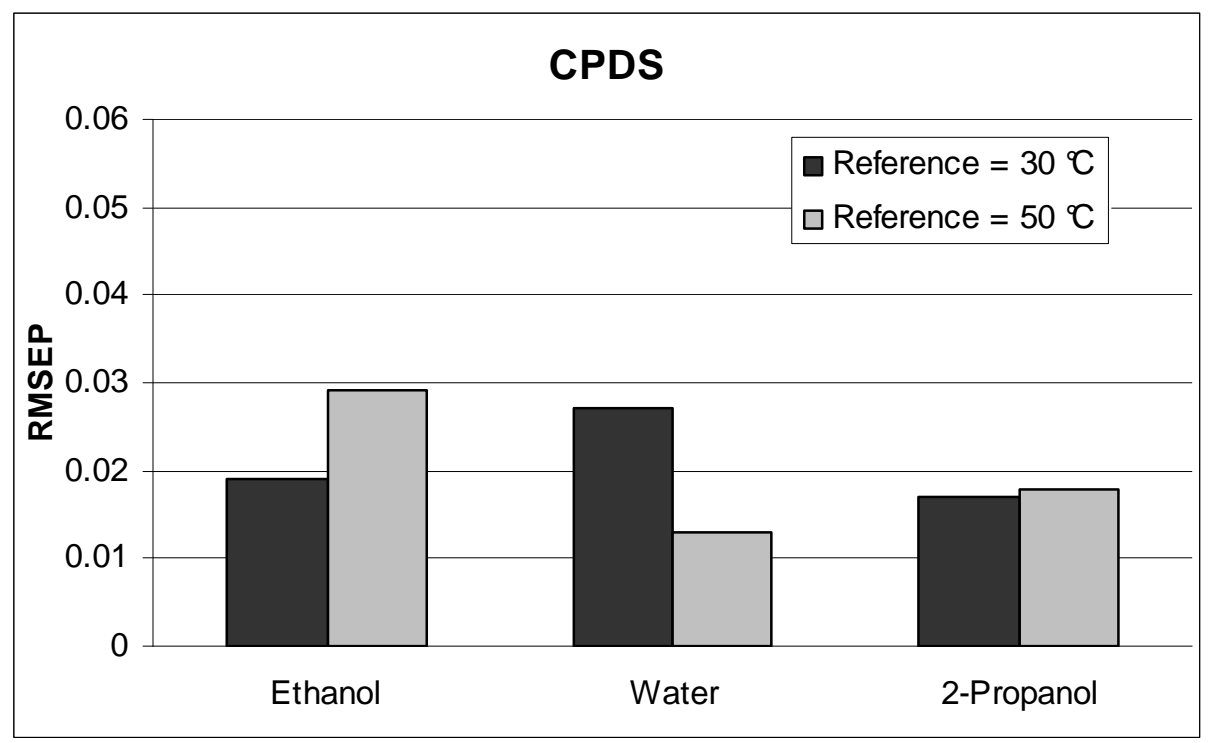

(b)

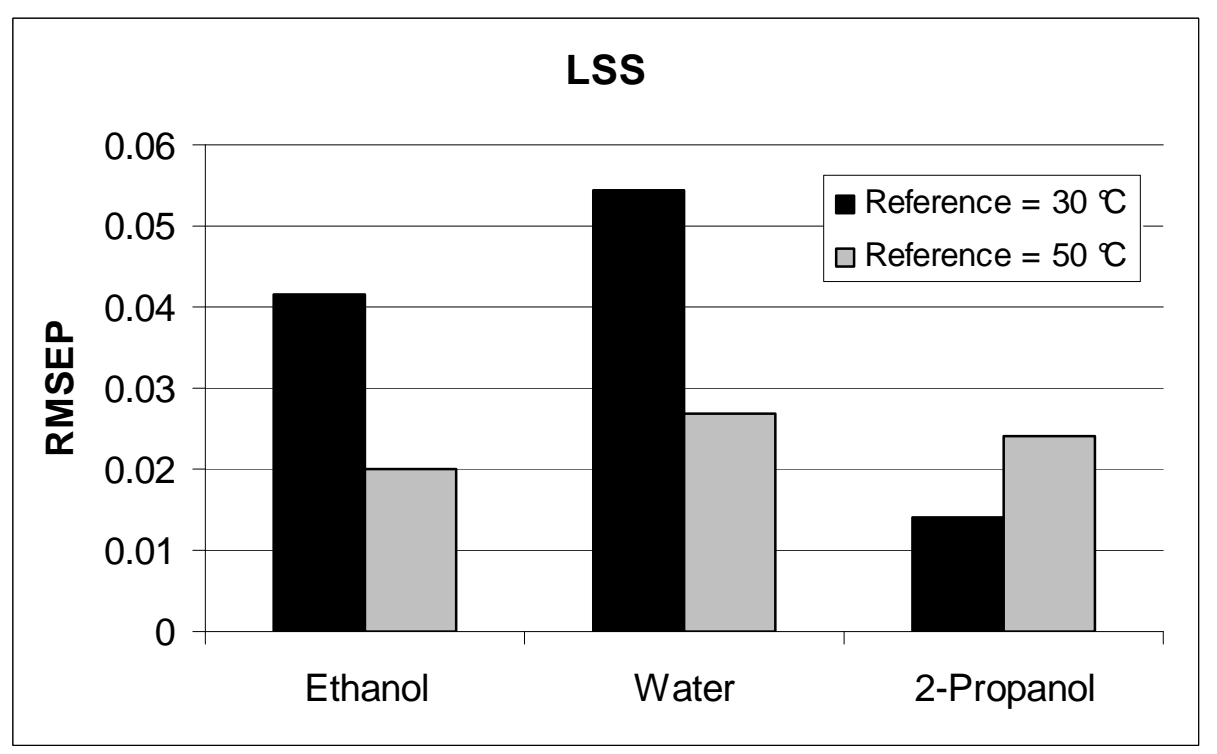


Figure 3: Predicted versus true LGA concentration (g/500ml water). RMSEPs: Global_1 (2.23); Global_3 (1.81).

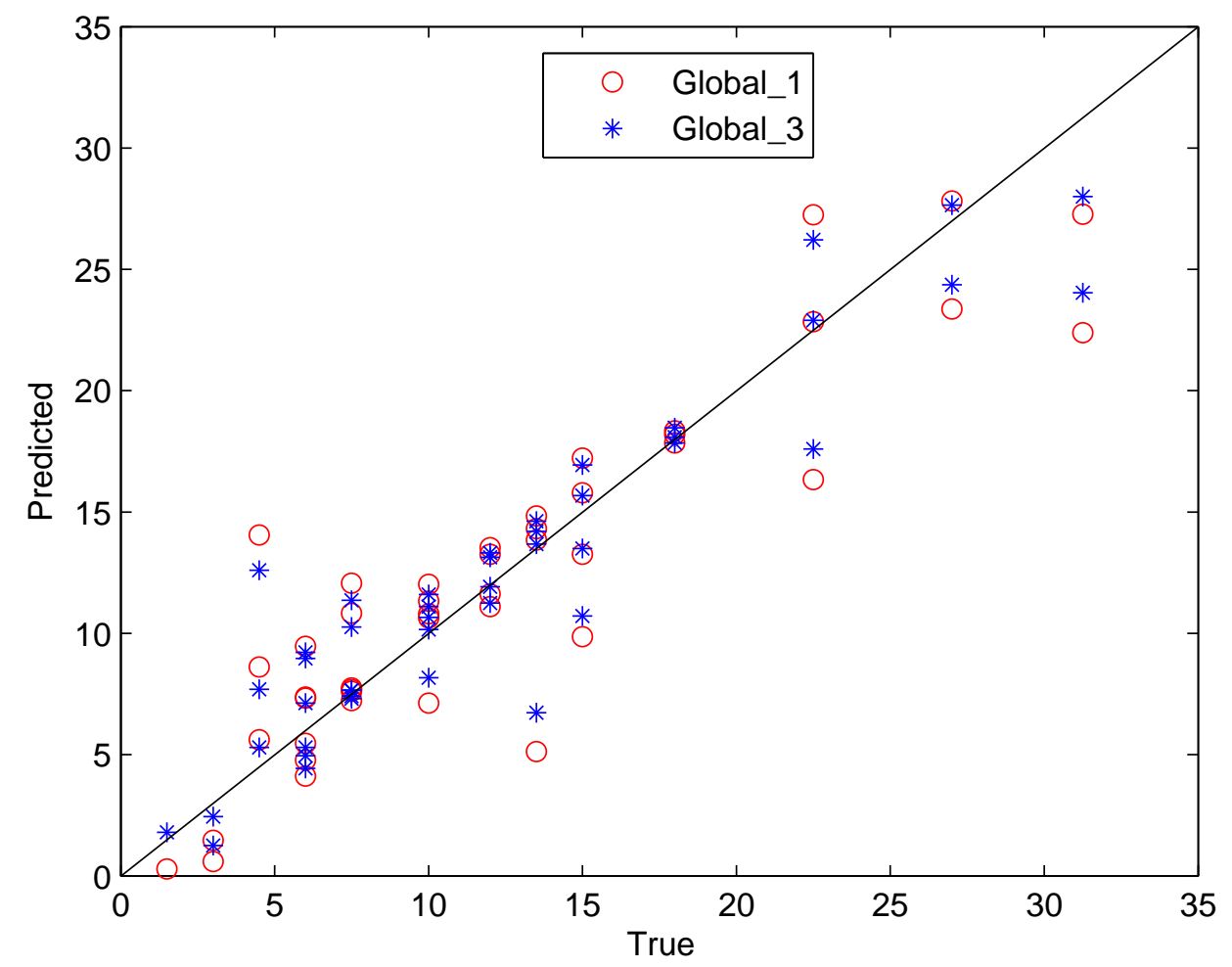

